\title{
Stereotype und Affektivität im interkulturellen Fremdsprachenunterricht
}

ABSTRACT. The following article discusses the relation between cultural stereotyping and affective processes in education leading to intercultural communication in modern languages studies. This work is both an attempt to explain this phenomenon theoretically and to research the previously mentioned relation in the light of postulates of intercultural education. A part of this work is also an analysis aiming at determining the factors influencing a stereotype category.

\section{EINLEITUNG}

Der vorliegende Artikel versteht sich als ein Beitrag zur fremdsprachendidaktischen Stereotypendiskussion mit besonderer Berücksichtigung der universitären Fremdsprachenvermittlung. Das Stereotyp ${ }^{1}$ als eine unumgängliche und stark affektiv unterbaute Kategorie der menschlichen Wahrnehmung nimmt einen zentralen Platz in dieser vor dem Hintergrund der Prämissen interkulturellen Fremdsprachenunterrichts stattfindenden Auseinandersetzung ein.

Der Forschungsbereich „nationale Stereotype im Fremdsprachenunterricht" kann eine Reihe von empirischen Befunden vorweisen (vgl. Bausinger

${ }^{1}$ In der Vielzahl von Arbeiten und Aufsätzen zu Stereotypen begegnet man unterschiedlichen Formen des Wortes Stereotyp; Es wird sowohl in der maskulinen als auch in der neutralen Form gebraucht. Auch wenn es um die Pluralform geht herrscht keine Einstimmigkeit. Zum einen sind es Stereotypen, zum anderen Stereotype. Nach Brockhaus Wahrig (Deutsches Wörterbuch in 6 Bänden, Stuttgart 1984) kann das Wort sowohl maskulin als auch neutral sein. Die Pluralform lässt auch beide Varianten zu. Laut Duden (Deutsches Universalwörterbuch, Mannheim 2001) ist das Wort Stereotyp n e u tra l und die Mehrzahl lautet S t e r e o t y pe. Die Duden-Version wird für den vorliegenden Beitrag übernommen. 
1988, 2000, Husemann 1990, 1991, Keller 1969b-1997, Löschmann 1998, Prokop 1995, Quasthoff 1986, 1989, Redder 1995, Reiß 1997, Rösch 2000, Zybatow 1994 u. a.). Paradoxerweise ist jedoch das Merkmal "Affektivität", welches Stereotype unzertrennlich begleitet, in der bisherigen Forschungspraxis kaum berücksichtigt worden. Man hat sich zumeist mit allgemeinen Tendenzen von Relationen zwischen den Variablen Stereotyp und Emotion beschäftigt.

Im Titel des vorliegenden Artikels werden Stereotype, Affektivität und interkultureller Fremdsprachenunterricht angesprochen. Warum werden gerade diese drei Konstrukte miteinander in Verbindung gesetzt? Es wird angenommen, dass diese drei Konstrukte eng verflochten sind. Es gibt kein interkulturelles Lernen ohne affektive Komponenten/Lernziele (KnappPotthoff 1997: 199ff., Tenberg 1999: 70f.) und die Auseinandersetzung mit Stereotypen (Krumm 2000b: 139). Laut Krumm (1995: 157, 2003b: 138ff.) verdankt sich der Stereotypen- und Vorurteilsforschung und den Versuchen, den Landeskundeunterricht in den Dienst der Überwindung von Vorurteilen und des Abbaus von Klischees zu stellen, ein wichtiger Impuls für die Entwicklung von Konzepten des interkulturellen Lernens. Weiterhin sind Stereotype auch als ein affektives Konstrukt zu verstehen (Quasthoff 1989: 43f., Roth 1989: 142), das vom interkulturellen Fremdsprachenunterricht nicht wegzudenken ist. Affektivität, verstanden vor allem als Emotionen und Einstellungen, die den Fremdsprachenlernprozess begleiten, ist besonders evident bei der Diskussion um Stereotype wahrzunehmen, welche ihrerseits im Rahmen der interkulturellen Themenschwerpunkte stattfindet.

\section{STEREOTYP UND AFFEKT - WIE NAH BEIEINANDER?}

Im Folgenden soll ein theoretischer und methodologischer Zusammenhang zwischen der Affektivität und Stereotypenbildung erläutert werden. Da die Problematik sowohl empirisch als auch theoretisch noch nicht ausgereift ist, soll sie auch an dieser Stelle nur ansatzweise dargestellt werden.

Stereotype fungieren auf drei verschiedenen Ebenen:

1) auf kognitiver Ebene,

2) auf sozialer Ebene,

3) auf politischer Ebene.

Nach Brunzel (2002: 86) ist in enger Verbindung mit der kognitiven Ebene außerdem auch eine emotionale Ebene zu postulieren, denn über Stereotype werden auch gefühlsmäßig Einstellungen zu unserer Umwelt, zu Menschen, Personen, Gegenständen, bestimmten Themen usw. transportiert. Stereotype geben uns eine emotionale Sicherheit: Sie schützen uns vor abträg- 
lichen Bildern und den damit verbundenen Gefühlen (Krampen 1990: 135). Roth (1989: 142) erklärt den Zusammenhang zwischen emotionaler und kognitiver Komponente im Stereotypisierungsprozess folgendermaßen:

"Ganz generell kann davon ausgegangen werden, dass die kognitive Tendenz zur Reduktion der Komplexität, zur Strukturierung, Akzentuierung und Polarisierung (...) mit der emotionaler Scheu vor zu großer Komplexität, zu viel Information, vor zu viel Neuem korrespondiert".

Stereotype sind für uns in emotionaler und kognitiver Hinsicht eminent wichtig, was ihre Langlebigkeit und ihre hohe Resistenz gegenüber Veränderungen erklären könnte:

"Ein Eingriff auf die Stereotype eines Individuums richtet sich gegen dessen psychologische (genauer: emotionale und kognitive) Basis" (Krampen 1990: 135).

Fiehler (1990: 46f.) bezeichnet Emotionen als bewertende Stellungnahmen und beschreibt sie mit einer konkreten Belegung als Formel: Emotion A ist eine bewertende Stellungnahme zu X auf der Grundlage von $Y$ als $Z$. Für $X, Y$ und $Z$ sind folgende Belegungen möglich (Tabl. 1)

Tabelle 1: Emotionen als Stellungnahmen (Fiehler 1990: 46f.)

\begin{tabular}{|c|c|c|}
\hline $\mathrm{zuX}$ & auf der Grundlage von $Y$ & als $\mathrm{Z}$ \\
\hline 1. Situation & 1. Erwartungen & 1. (gut) entsprechend \\
\hline 2. andere Person & 2. Interessen, Wünsche & 2. nicht entsprechend \\
\hline 3. eigene Peson & 3. (akzeptierte) soziale Nor- & \\
\hline (Handlung, Eigenschaft) & men/Moralvorstellungen & \\
\hline 4. Ereignis/Sachverhalt & 4. Selbstbild & \\
\hline 5. Gegenstände & 5. Bild des anderen & \\
\hline 6. mentale Produktionen & & \\
\hline
\end{tabular}

Fiehler (ebd.) illustriert sein Konzept folgendermaßen:

„Ärgere ich mich, weil ich eine Vase umgestoßen habe, so lässt sich dies auffassen als bewertende Stellungnahme zu der eigenen Person (bzw. einer Aktivität von ihr) auf der Grundlage des Selbstbildes (bzw. Erwartungen über mein Verhalten) als nicht entsprechend. Freue ich mich bei dem Gedanken, dass ich morgen Besuch bekommen werde, so ist dies beschreibbar als bewertende Stellungnahme auf der Grundlage meiner Wünsche (oder Erwartungen) zu einer mentalen Produktion als gut entsprechend".

In dieser Konzeptualisierung und Definition von Emotion spielen Selbstund Fremdbilder (Auto- und Heterostereotype) eine nicht zu überschätzende Rolle. Sie sind Grundlage einer emotionalen Stellungnahme zu bestimmten Situationen, Personen, Ereignissen...etc. 
Für Quasthoff (1989: 43f.) ist die affektive Funktion der Stereotype in Verbindung mit der sog. autoritären Persönlichkeit, die von Adorno in den 40er Jahren entwickelt wurde, zu verstehen. 1954 bestimmte Allport Stereotype als Rationalisierungen entsprechender Einstellungen. Demnach sei die affektive Einstellung (Ich mag keine X) das psychisch Primäre.

Nach dem Drei-Komponenten-Ansatz ${ }^{2}$, der sowohl für die Einstellung als auch für das Stereotyp anzuwenden ist, bestehen beide Konstrukte aus drei Komponenten: einer kognitiven, affektiven und konativen Komponente. Aus den Äußerungen der Lernenden, in denen sie rückblickend Erlebnisse, Erfahrungen oder allgemeine Sachverhalte darstellen, können mithilfe dieses Modells Stereotype herausgearbeitet werden, indem die oben genannten Komponenten als Basis herangezogen werden, auf denen ein Stereotyp (bzw. eine Einstellung) beruht.

Einstellungen sind nicht direkt messbar, sondern nur über MeinungsäuBerungen zugänglich (Quasthoff 1973: 27). Stereotype werden demnach als Meinungsäußerungen verstanden. Es werden von uns also Stereotype untersucht, die aus Einstellungen resultieren, wie es die folgende Abbildung 1. illustriert:

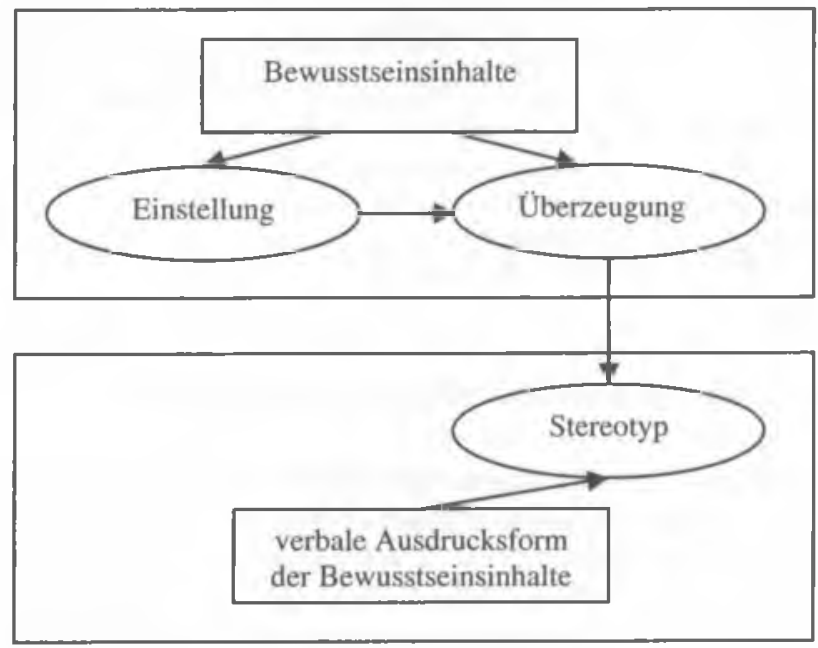

Abb. 1: Das Stereotyp, Einstellung und Überzeugung (Bearbeitung M.P.)

${ }^{2}$ Anders: mehrdimensionale Einstellungskonzeption. Dieses Modell setzte sich seit Anfang der 60er Jahre durch. Mit der affektiven Komponente ist - gefuihlsbestimmte Bewertung eines Objekts, mit der kognitiven - Inhalt und Struktur des Wissens, das zu dem Einstellungsobjekt repräsentiert wird und mit der konativen Komponente - beobachtbares, mit einer Einstellung verknüpfbares Handeln, gemeint (Seel 2000: 119). 
Kommen wir jedoch $\mathrm{zu}$ fremdsprachendidaktischen Implikationen des Zusammenhangs zwischen Einstellung und Stereotyp. Im Falle der Wahrnehmung einer Fremdkultur kommt es nach Keller (1994: 82f.) in mentalen Verstehensprozessen zur Auswahl der als wichtig angesehenen Fakten und zur Schematisierung, die in Stereotypen ihren Niederschlag findet. Dabei wird die Wahrnehmung einer Fremdkultur im Gegensatz zu einer als unvoreingenommen geltenden Beobachtung durch kognitive und affektive Perspektiven gesteuert, die weniger zu alten Ego-Spiegelungen als vielmehr zu Verzerrungen der Fremdkultur führen.

Affektive Wertung ist eine unumgängliche Komponente des Kulturvergleichs, der wiederum den Fremdsprachenlernprozess immer begleitet (Keller 1998: 152f.). Erfahrungen aus der eigenen Kultur mit ihren Wertvorstellungen, Verhaltensweisen und soziokulturellen Errungenschaften dienen als Beurteilungskriterien für eine Orientierung in der Fremdkultur. Dabei werden u.a. Bevölkerungszahl, Klima, Wirtschaft, Einkommensverhältnisse, Sozial- und Ausbildungssysteme, Lebensstandart, Wertvorstellungen und Verhaltensweisen miteinander verglichen und einer affektiven Wertung unterzogen, wobei sich Ähnlichkeiten und Unterschiede mit der eigenen Kultur herauskristallisieren. Dabei werden die stereotypen Vorstellungen vom Ausland in ein kausales, funktionales, emotionales und selbstreferentielles Beziehungsgeflecht eingebettet, das als Orientierungsraster für das Fremdverstehen dient.

Aufgrund dieser Erkenntnisse wird für eine bewusste Auseinandersetzung mit Einstellungen und Stereotypen als motivations- und emotionsauslösenden Dispositionen und eigener Sprach- und Kulturbedingtheit im Fremdsprachenunterricht plädiert.

\section{STEREOTYP UND AFFEKTIVITÄT IN DER HOLISTISCHEN BETRACHTUNG ALLER DETERMINANTEN DES FREMDSPRACHENLERNPROZESSES}

Das Konzept des vorliegenden Beitrags, das kulturelle Stereotypie und Affektivität theoretisch zu verbinden sucht, wird anhand der folgenden Abbildung illustriert (Abb. 2): Das Stereotyp wird danach als eine Disposition verstanden, die stark im affektiven Bereich angesiedelt ist, und der Motivation, Interesse und Emotionen und Einstellungen zugrunde liegen. Einstellungen und Überzeugungen werden nach Quasthoff (1973) als Bewusstseinsinhalte verstanden, Stereotype dagegen als ihre verbalisierten Ausdrucksformen. Diese Disposition korreliert ebenfalls mit vielen anderen biographiebedingten Faktoren, die sich allgemein in drei Gruppen einteilen lassen: persönlichkeitsspezifische Faktoren, Fremdsprachenunterricht und soziale Umwelt. 


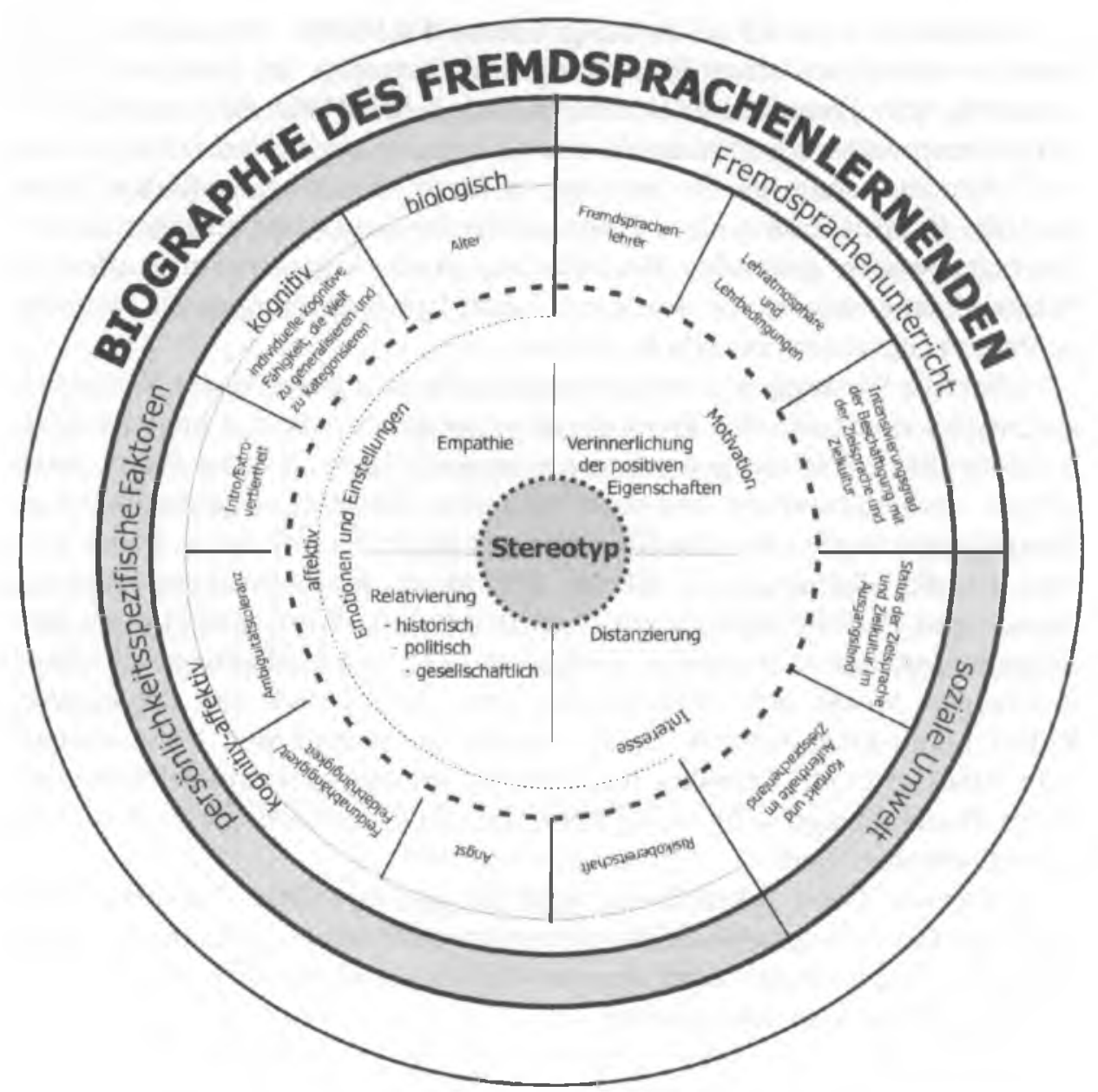

Abb. 2: Stereotyp und Affektivität in der holistischen Betrachtung aller Determinanten des Fremdsprachenlernprozesses (Bearbeitung von M.P.)

Unter persönlichkeitsspezifischen Faktoren werden biologische, kognitive, kognitiv-affektive und affektive Variablen verstanden, die sich weiter in mehrere Variablen einteilen lassen. Zur Faktorenkomplexion Fremdsprachenunterricht gehören: der Fremdsprachenlehrer, Lehratmosphäre und Lehrbedingungen, und der Intensivierungsgrad der Beschäftigung mit der Zielsprache und Zielkultur. Die soziale Umwelt wird als Status der Zielsprache und -kultur im Ausgangsland und Kontakt und Aufenthalte im Zielsprachenland verstanden.

Darüber hinaus wird das Stereotyp als eine aktive Kategorie verstanden, die immer wieder neu erschaffen, gehandelt, verstanden und individuell 
wahrgenommen und vertreten wird: Stereotype können relativiert werden; man kann sich von ihnen distanzieren; eventuell in sie einzufühlen versuchen (Empathie); und auch die stereotypen Eigenschaften verinnerlichen wollen.

In diesem Zusammenhang wird die Frage gestellt, inwieweit sich die Beschäftigung mit dem Stereotyp in die Prämissen des interkulturellen Lernens fügt. Welche Eigenschaften, welche Voraussetzungen oder welche Charakteristika sind erforderlich für interkulturelles Lernen? Inwieweit wird die Auseinandersetzung mit dem Stereotyp von diesen Charakteristika bedingt?

Wenn wir einige Groblernziele des interkulturellen Lernens anführen, die sich entlang einer heuristischen Differenzierung in affektive, kognitive und handlungsorientierte Komponenten einteilen lassen (z. B. Tenberg 1999: 70f., Kaikkonen 1997: 78ff.), stellen wir fest, dass sich die Zielsetzungen des interkulturellen Lernens und der Beschäftigung mit dem Stereotyp als einer kognitiv-affektiv-konativen Kategorie größtenteils decken. Dementsprechend lassen sich Lernziele des fremdsprachenunterrichtlichen Umgangs mit Stereotypen im Blickwinkel der Interkulturalität folgend formulieren:

1. affektiv: Reflexion über Auto- und Heterostereotype $\rightarrow$ Selbst- und Fremdbilder $\rightarrow$ Eigen- und Fremdkultur $\rightarrow$ Sensibilisierung für einen interkulturellen Sichtwechsel $\rightarrow$ Vollzug einer Veränderung der affektiven Einstellung gegenüber Eigen- und Fremdkulturellem.

Solche Merkmale wie Non-Ethnozentrismus, Toleranz, Empathie, Rollenflexibilität, Respekt und Wertschätzung für die fremde Kultur, nicht-wertender Attributionsstil und Ambiquitätstoleranz lassen sich dann mühelos als postulierbare outcomes einordnen;

2. kognitiv: Erwerb von Kenntnissen und Einsichten über:

a. diachronische und synchronische Beziehungen von Auto- und Heterostereotypen $\rightarrow$ Selbst- und Fremdbildern $\rightarrow$ Eigen- und Fremdkultur,

b. die "kollektiven Wissensvorräte" (einschließlich des Stereotypenrepertoires) im Hinblick auf eigene und fremde Perspektiven und diese in ihrer historischen Abhängigkeit erkennen,

c. soziale Interaktionsformen und - rituale in der Fremdkultur;

3. handlungsorientiert: Sensibilisierung für und Kenntnisse über eigene und fremdkulturelle Wissensvorräte (einschließlich der Auto- und Heterostereotype) sind Grundlagen für handlungsorientierte Lernziele (Entwicklung von Investigations-, Interpretations- und Relationsfertigkeiten).

Zusammenfassend wird festgestellt, dass im interkulturellen Fremdsprachenunterricht, der sowohl die affektive und als auch kognitive Seite des Lernens anvisiert, ein entsprechender Umgang mit Stereotypen nicht wegzudenken ist. 


\section{STEREOTYP UND AFFEKT IM INTERKULTURELLEN FREMDSPRACHENUNTERRICHT - EIN PRAXISORIENTIERTES MODELL}

Den Weg zur interkulturellen Kommunikation teilen wir in drei Phasen: ${ }^{3}$

1) Wahrnehmung,

2) Bedeutungsentwicklung,

3) Kommunikation.

Dabei platzieren wir Stereotypie und die den Stereotypisierungsprozess unzertrennlich begleitende Affektivität in die Wahrnehmungsphase und behaupten mit Krumm (1994: 122), dass „....interkultureller Fremdsprachenunterricht darauf abzielen sollte, ein systematisches Wahrnehmungstraining zu entwickeln, das kulturgebundene Deutungsmuster in der Sprache aufsucht und Prozesse des Selbst- und Fremdverstehens in den Mittelpunkt rückt". Stereotype verstehen wir in diesem Zusammenhang als kulturgebundene Deutungsmuster. Die Auseinandersetzung mit ihnen ist für Prozesse des Selbst- und Fremdverstehens unumgänglich. Wir plädieren also für eine Wahrnehmungsschulung, welche reflektierten Umgang mit Stereotypen einbezieht.

Folgende unterrichtliche Tätigkeiten und didaktische Entscheidungen sollen zur Wahrnehmungsschulung im interkulturell ausgerichteten Fremdsprachenunterricht, der den Umgang mit der Kategorie des Stereotyps berücksichtigt, bevorzugt werden:

1. Sensibilisierung für Stereotype durch Gespräche, Filme, authentische Texte (gesteuertes interkulturelles Lernen);

2. Arbeit mit authentischen Texten, Tonbändern, Bildmaterialien und Filmen (ungesteuertes interkulturelles Lernen);

3. Simulationen und simulative Übungen;

4. Projektarbeit mit soziokulturellen bzw. landeskundlichen Themen einschließlich der Stereotype (Familie, Wohnen, Umwelt, Verkehr usw.);

5. Ausarbeitung und Übernahme einer fremden Rolle;

6. Muttersprachler mit der interkulturellen Kompetenz und jeweiligen Kenntnissen als Ansprechpartner;

7. Förderung von Studienfahrten mit einem intensiven interkulturellen Programm und von 1 bis 2 semesterlangen Ziellandsaufenthalten.

Bezüglich dieses letzten Postulats soll hervorgehoben werden, dass die interkulturelle Kompetenz nicht "trocken" geübt werden kann. Aus diesem

${ }^{3}$ Diese Einteilung haben wir Sendzik und Rahlwes (1988) übernommen. Erwähnenswert ist ebenfalls das taxonomische Modell kognitiver Handlungsebenen von Bloom (1976, auch Myczko 2002: 72), welches sich aus folgenden Ebenen zusammensetzt: Evaluation, Analyse, Verstehen und Wiedererkennen. Die Ebene der Evaluation umfasst die Wahrnehmung und Bewertung, welche wiederum solche Kategorien wie Stereotyp und Affekt aufweist. 
Grunde betonen wir wiederholt die Relevanz von Studienfahrten, Austauschprogrammen und Ziellandsaufenthalten. Ihnen soll aber eine entsprechende interkulturelle Vorbereitung vorausgehen. Wir sind uns im Klaren, dass dies ein hochgestecktes Ziel ist - aber hoffentlich kein unerreichbares bleiben wird. In den letzten zehn Jahren hat sich die Zahl der an Austauschprogrammen teilnehmenden Studenten sowohl in Deutschland als auch in Polen um ein Vielfaches erhöht.

Darüber hinaus sollen im interkulturellen Fremdsprachenunterricht dem Lernenden Bedingungen geschaffen werden, welche ihm eine Explizierung der subjektiven Theorien zu Stereotypen wie auch eine Metareflexion ermöglichen würden. Im universitären Bereich, besonders im Philologiestudium soll eine solche Explizierung und Auseinandersetzung mit dem Stereotyp vor allem im Rahmen der sprachpraktischen Übungen stattfinden. Da eine solche Auseinandersetzung stark affektiv geprägt ist, d. h. eigene Erfahrungen, Erlebnisse und Sichtweisen mit einbezogen und ausdiskutiert werden, und da sie die sog. Alltagskultur betrifft, eignet sich der sprachpraktische Unterricht, der meistens einen lockeren Stil und Inhalt hat, am besten dazu. Die Thematik der Stereotype ist für Philologiestudenten äußerst interessant. Sie begleitet sie und reift während des gesamten Studiums und auch später. Jeder interkulturelle Kontakt bringt eine Auseinandersetzung mit den eigenen Selbst- und Fremdbildern mit sich. So gesehen ist eine solche Explizierung der subjektiven Theorien zu Stereotypen gerechtfertigt und empfehlenswert.

Ebenfalls relevant scheint die Beschäftigung mit Prinzipien allgemeiner Semantik zu sein, welche parallel zur Wahrnehmungsschulung und zur Explizierung der subjektiven Theorien zu Stereotypen stattfinden soll. Die Lernenden sollen die Möglichkeit haben, zu erkennen, welche Rolle Sprache für menschliches Verhalten spielt und wie sie dieses beeinflusst. Dabei sollen tiefgehende Zusammenhänge zwischen Sprache, Kultur, Zivilisation und kulturbedingter Fremdwahrnehmung erkannt werden. Durch bewusste Sprachbetrachtung und Analyse eigenen Sprachverhaltens sollen sie mehr über eigene Kognitionen erfahren und entdecken, dass Wort und Gegenstand nicht übereinstimmen, dass gleiche Dinge in verschiedenen Sprachen unterschiedliche Bezeichnungen besitzen, Sprache und Verhaltensweisen sich ständig wandeln und jeder Mensch, je nach Standpunkt und Interessen, seine Umgebung unterschiedlich wahrnimmt, sodass Situationsbeschreibungen zugleich Tatsachen und deren Wertungen beinhalten und insofern ein Abbild davon geben, was im Betrachter selbst vorgeht.

Die folgende Abbildung 3 stellt eine Zusammenfassung und zugleich einen Vorschlag holistisch gesehenen Umgangs mit der Kategorie Stereotyp im interkulturellen Fremdsprachenunterricht dar: 


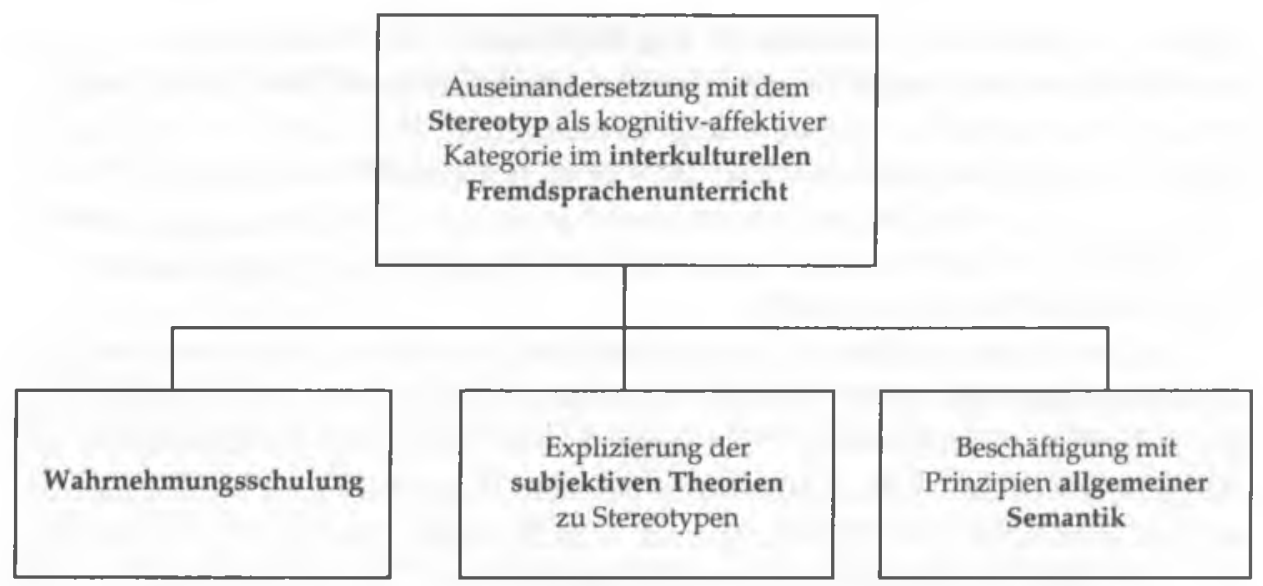

Abb. 3: Holistischer Umgang mit dem Stereotyp im interkulturellen Fremdsprachenunterricht (Bearbeitung M.P.)

Man soll dabei nicht vergessen, dass die Erziehung zur interkulturellen Kompetenz und Kommunikation ein prozess- und nicht produktorientiertes Lernen ist. So muss man auch die Auseinandersetzung mit dem Stereotyp prozessorientiert betrachten und dementsprechend didaktisch gestalten. Ebenfalls wichtig scheint uns ein systematisches didaktisches Vorgehen zu sein, dem eine konzentrierte (also nicht lineare) Darbietung des Lernstoffes zugrunde liegt.

Wenn man bedenkt, dass die interkulturelle Kompetenz durch drei Begriffe: Kenntnisse, Motivation und Handeln (Zawadzka 2004a: 213) bedingt ist, soll verstärkt für die Berücksichtigung aller drei Komponenten in der Schulung dieser Kompetenz in Bezug auf den Umgang mit der Kategorie des Stereotyps plädiert werden. Folgende drei Fragestellungen sind besonders relevant:

1. Welche Kenntnisse um die Kategorie des Stereotyps sind nützlich für interkulturelle Kommunikation und wie werden sie erworben?

2. Wie kann man für die Fremdsprachenlernenden eine solche Motivationsbasis schaffen, dass sie sich bemühen, in Richtung der Zielvorstellungen des Umgangs mit der Kategorie des Stereotyps zu arbeiten und sich die interkulturelle Kompetenz zu erwerben?

3. Durch welche (hochschulischen) Maßnahmen kommt man zu einem interkulturellen und interkulturell geprägten Handeln in Bezug auf den Umgang mit der Kategorie des Stereotyps?

Die Problemstellung unserer fremdsprachlichen Unterrichtstätigkeit liegt also darin, ob und inwiefern sowohl Relativierung und Differenzierung von 
Stereotypen als auch Eigen- und Fremdverstehen im hochschulischen Fremdsprachenunterricht außerhalb der Zielsprachenkultur möglich sind. Eine relevante Frage besteht darin, ob durch hochschulische Maßnahmen solche Bedingungen geschaffen werden können, die den Lernenden sowohl zu einer zufriedenstellenden fremdsprachlichen Kompetenz als auch zum interkulturellen Lernen und Stereotypenrelativierung führen.

Übergreifende Lernziele in der Auseinandersetzung mit der Kategorie Stereotyp auf dem Wege zur Schulung der interkulturellen Kompetenz sind:

1. Einsichten in die (linguistische) Struktur des Stereotyps zu erarbeiten;

2. Eigene und fremde Stereotype als solche zu erkennen und ihren kognitiven, affektiven und konativen Wert zu berücksichtigen. Wenn sie in verdeckter, impliziter Form erscheinen, sind dazu bestimmte Analyseverfahren erforderlich;

3. Die Entstehung von Stereotypen und ihren Erwerb durch das Individuum zu reflektieren;

4. Einsichten in die Funktion von Stereotypen für das Individuum und für die Gruppe/Gesellschaft zu gewinnen;

5. Die grundsätzliche Bereitschaft zu entwickeln, Stereotype in den Situationen, in denen eine Konfrontation mit dem stereotypisierten Objekt ansteht, auf ihren Gehalt hin zu prüfen.

Die vorliegenden Ausführungen sollen zu einem aufgeklärten Umgang mit Stereotypen im Fremdsprachenunterricht anregen. Die ermittelten Stereotype und der sie unzertrennlich begleitende affektive Wert weisen auf die Notwendigkeit hin, dem Stereotyp einen angemessenen Platz im interkulturellen Fremdsprachenunterricht, der ein soziales, kognitives und affektives Lernen voraussetzt, zu gewähren.

\section{BIBLIOGRAPHIE}

Bausinger, H., 1988. Stereotypie und Wirklichkeit. In: Jahrbuch Deutsch als Fremdsprache, 14, München: 157-170.

Bausinger, H., 2000. Typisch deutsch. Wie deutsch sind die Deutschen? München: Beck.

Bloom, B. S. u. a., 1976. Taxonomie von Lernzielen im kognitiven Bereich. Weinheim/Basel: Beltz.

Brunzel, P., 2002. Kulturbezogenes Lernen und Interkulturalität. Zur Entwicklung kultureller Konnotationen im Französischunterricht der Sekundarstufe I. Tübingen: Gunter Narr Verlag.

Düwell, H., 2003. Fremdsprachenlerner. In: Bausch / Christ / Krumm 2003: 347-352.

Fiehler, R., 1990. Kommunikation und Emotion. Theoretische und empirische Untersuchungen zur Rolle von Emotionen in der verbalen Interaktion. Berlin/New York: Walter de Gruyter.

Husemann, H., 1990. Stereotypen in der Landeskunde. In: Neusprachliche Mitteilungen aus Wissenschaft und Praxis, 2: 89-98.

Husemann, H., 1991. Stereotypes in Landeskunde - shall we join them if we cannot beat them? In: (Hg.) Mediating a Foreign Culture: The United States and Germany. L. Bredella, Tübingen: 17-35. 
Kaikkonen, P., 1997. Fremdverstehen durch schulischen Fremdsprachenunterricht. In: Info $D a F, 1: 78-86$.

Keller, G., 1969b. Die Funktion von Stereotypen beim Erkenntnisprozess im kulturkundlichen Unterricht - dargestellt an einer Strukturanalyse von Schülerurteilen. In: Die Neueren Sprachen, 68: 175-186.

Keller, G., 1970. Die Änderung kognitiver Urteilsstrukturen durch einen Auslandsaufenthalt. In: Praxis, 4: 352-374.

Keller, G., 1983. Grundlegung einer neuen Kulturkunde als Orientierungsrahmen für Lehrerausbildung und Unterrichtspraxis. In: Neusprachliche Mitteilungen, 4: 200-210.

Keller, G., 1987. Auto- und Heterostereotype amerikanischer und deutscher Schüler in einer neuen Kulturkunde. In: Die Neueren Sprachen, 1: 63-79.

Keller, G., 1994. Interkulturelles Lernen aus der Perspektive von Systemtheorie und Sozialpsychologie. In: Neusprachliche Mitteilungen aus Wissenschaft und Praxis, 2: 81-85.

Keller, G., 1997. Welcher Stellenwert sollte dem Image der (hässlichen?) Deutschen im Fremdsprachenunterricht zukommen? In: Neusprachliche Mitteilungen aus Wissenschaft und Praxis, 2: $90-93$.

Keller, G., 1998. Didaktik des Fremdverstehens aus neurobiologisch-konstruktivistischer empiricher Sicht. In: Löschmann M. und Stroinska M. (Hg.), Stereotype im Fremdsprachenunterricht. Frankfurt am Main: 143-160.

Knapp-Potthoff, A., 1997. Interkulturelle Kommunikationsfähigkeit als Lernziel. In: KnappPotthoff A. und Liedke M. (Hg.), Aspekte interkultureller Kommunikationsfähigkeit. München: Iudicium: 181-205.

Krampen, M., 1990. Stereotypen von Architekturvorstellungen. In: Koch W. (Hg.), Natürlichkeit der Sprache und Kultur: acta colloquii. Bochum: Brockmeyer: 134-151.

Krumm, H.-J., 1995. Interkulturelles Lernen und interkulturelle Kommunikation. In: Bausch / Christ / Krumm (Hg.), Handbuch Fremdsprachenunterricht. Tübingen: Francke: 151-161.

Krumm, H.-J., 2003b. Curriculare Aspekte des interkulturellen Lernens und der interkulturellen Kommunikation. In: Bausch / Christ / Krumm (Hg.), Handbuch Fremdsprachenunterricht. Tübingen: Francke: 138-144.

Löschmann, M., 1998. Stereotype, Stereotype und kein Ende. In: Löschmann M./Stroinska M. (Hg.), Stereotype im Fremdsprachenunterricht, Frankfurt a. M. 7-34.

Myczko, K., 2002. Kognition und interkulturelle Kompetenz. In: T. Siek-Piskozub (Hg.) European year of languages 2001. Poznan: UAM; 69-74.

Prokop, I., 1995. Stereotype, Fremdbilder und Vorurteile. In: Czyżewski/Gülich/Hausendorf/Kastner, Nationale Selbst- und Fremdbilder im Gespräch., Westdeutscher Verlag, Opladen: 180-202.

Putnam, H., 1979. Die Bedeutung von „Bedeutung”. Frankfurt a. M.: Vittorio Klostermann.

Quasthoff, U. M., 1973. Soziales Vorurteil und Kommunikation - Eine Sprachwissenschaftliche Analyse des Stereotyps. Frankfurt a. M.: Athenäum Verlag.

Quasthoff, U. M., 1986. Nichtsprachliches und "semisprachliches" Wissen in interkultureller Kommunikation und Fremdsprachendidaktik. In: Die Neueren Sprachen, 3: 230-253.

Quasthoff, U. M., 1989. Ethnozentrische Verarbeitung von Informationen: Zur Ambivalenz der Funktionen von Stereotypen in der interkulturellen Kommunikation. In: P. Matusche (Hg.) Wie verstehen wir Fremdes? Aspekte zur Klärung von Verstehensprozessen. Dokumentation eines Werkstattgesprächs des Goethe-Institut München vom 24.-26. November 1988. München: 37-62.

Redder, A., 1995. "Stereotyp" - eine sprachwissenschaftliche Kritik. In: Jahrbuch Deutsch als Fremdsprache, 21: 311-329.

Reiß, S., 1997. Stereotypen und Fremdsprachendidaktik. Hamburg: Kovac.

Riemer, C., 1997. Individuelle Unterschiede im Fremdsprachenerwerb. Hohengehren: Schneider Verlag. 
Riemer, C./Schlak, T., 2004. Der Faktor Motivation in der Fremdsprachenforschung. Einleitung in das Themenheft. In: Zeitschrift für Interkulturellen Fremdsprachenunterricht [Online], http://www.ualberta.ca/ german/ejournal/Riemer_Schlak2.htm, 9(2).

Rösch, O., 2000. Alles beim Alten? Ergebnisse einer Befragung polnischer und deutscher Studenten. In: Rösch O. (Hg.), Stereotypisierung des Fremden. Auswirkungen in der Kommunikation. Berlin, 207-223.

Roth, W., 1989. Vorerfahrungen und Stereotypien: Thre Veränderbarkeit durch zweisprachige Kontakte? In: Pelz M. (Hg.), Lerne die Sprache des Nachbarn. Grenzüberschreitende Spracharbeit zwischen Deutschland und Frankreich. Frankfurt a. M.: Diesterweg: 134-149.

Seel, N: M., 2000. Psychologie des Lernens. Lehrbuch für Pädagogen und Psychologen. München: Ernst Reinhardt Verlag.

Sendzik, J./Rahlwes, S., 1988. Lernort Frankreich: Schüleraustausch als praktisches Lernen. In: Edelhoff C./Liebau, E. (Hg.). Über die Grenze. Praktisches Lernen im Fremdsprachenunterricht. Weinheim und Basel: Beltz.

Tenberg, R., 1999. Theorie und Praxis bei der Vermittlung von, interkulturellen Kompetenzen'. In: Bredella L. / Delanoy (Hg.), Interkultureller Fremdsprachenunterricht. Tübingen, 65-85.

Zawadzka, E., 2004a. Nauczyciele jezzyków obcych w dobie przemian. Kraków: Oficyna Wydawnicza "Impuls".

Zawadzka, E., 2004b. Selbst- und Fremdbilder - nur ein fremddidaktisches Problem? In: Badstübner-Kizik C. / Rozwalowska-Żądło R., Uniszewska A., Sprachen lehren - Sprachen lernen. Nauczanie i uczenie się języków obcych. Gdansk: Wydawnictwo Uniwersytetu Gdanskiego, 331-345.

Zybatow, L., 1994. Sprachstereotyp, Denkstereotyp und interkulturelle Kommunikation. In: Beckmann S. / König P.-P. (Hg.), Satz - Text - Diskurs. Akten des 27. Linguistischen Kolloquiums. Münster 1992. Tübingen: 307-321. 